УДК 164.07

DOI:

10.15372/PS20150305

\title{
С.Е. Овчинников
}

Институт философии и права СО РАН, г.Новосибирск

step.ovch@gmail.com

\section{ФИЛОСОФСКАЯ ЗНАЧИМОСТЬ РЕДУКТИВНЫХ И НЕРЕДУКТИВНЫХ ПОДХОДОВ К МОДЕЛИРОВАНИЮ СОЗНАНИЯ"}

В статье рассматриваются философские основания современных способов понимания и моделирования сознания. Выделяются внутренние противоречия, проводится анализ взаимной критики редуктивных и нередуктивных направлений. Определена основная причина разногласия между концепциями и философские следствия из нее.

Ключевые слова: сознание, редукция, супервентность, квалиа, субстанция, феномен.

\section{S.E. Ovchinnikov}

Institute of Philosophy and Law SB RAS, Novosibirsk

step.ovch@gmail.com

\section{PHILOSOPHICAL SIGNIFICANCE OF REDUCTIVE AND NON-REDUCTIVE APPROACHES TO THE SIMULATION OF THE CONSCIOUSNESS}

The paper consider philosophical foundation of modern approach to understand and model of consciousness. The inner contradictions are distinguished, analyzes of relative critics of reductive and non-reductive approaches is developed. The main cause of disagreement between conceptions is determinate and philosophical consequences from it are deduced.

Keywods: consciousness, reduction, supervenience, qualia, substance, phenomenon.

* Публикуется в авторской редакции

(c) Овчинников С.Е., 2015 
Феномен сознания является одной из центральных тем современной философии и науки. На текущий момент существует множество различных конкурирующих теорий, но ни одна из них не способна привлечь всех исследователей под свои флаги. Все концепции, так или иначе, затрагивают общие вопросы о природе и роли сознания в мире:

1) Существует ли особая, отличная от материи, субстанция для сознания?

Перед исследователями, отвечающими на этот вопрос положительно, ставится следующий:

2) Каким образом осуществляется причинная связь между материальным телом, и нематериальным сознанием?

Отрицательный ответ на первый вопрос так же влечет последствия:

3) Каким образом можно описать сознание в физических терминах?

Ответ на первый вопрос определяет онтологию теории сознания и задает основное направление исследования. Все теории можно расположить на отрезке между числами 0 и 1 , в зависимости от ответа на онтологический вопрос, где 0 символизирует однозначное «нет», а 1 «да» (редуктивный физикализм - супервентный физикализм - функционализм - дуализм свойств - дуализм). Редуктивный подход предполагает выведение свойств сознания из его физического носителя, нередуктивный утверждает принципиальную невозможность такого вывода по тем или иным причинам.

Одной из причин данного разногласия, как кажется, является различие в определениях сознания. Как следствие в литературе нет ни удовлетворительного определения сознания (такого, которое бы удовлетворяло всех) ни единой теории.

Сторонники крайних физикалистских концепций, как правило, опираются на классические материалистически установки. Материальный мир каузально замкнут, никакой другой субстанции не существует.

С точки зрения редукционистов абсолютным авторитетом в области построения картины мира является физическая наука. Явления, изучаемые физикой, признаются за базисные. Они, во-первых, не требуют дополнительного объяснения (т.е. редукции к чему-либо более фундаментальному), во-вторых, из них можно выводить все остальные факты о мире. Из физических фактов выводятся химические, из химических, 
в свою очередь, биологические. Далее из биологических должны выводится социальные или сознательные факты, но современная наука не дает полноценного вывода. Тем не менее, уповая на опыт уже имеющихся успехов редукции, многие исследователи аппроксимируют процесс и считают, что такой вывод принципиально возможен. Данный подход подразумевает объективизм и «позицию от третьего лица» в исследовании сознания.

В зависимости от сферы интересов исследователя, сознание редуцируется либо к физическим фактам (редуктивный физикализм), либо к биологическим (нейрофилософия), либо к когнитивным (функционализм).

Для ответа на вопрос о редукции требуется три элемента:

1) Что редуцировать?

2) К чему редуцировать?

3) Как редуцировать?

Как правило, каждый подход обладает ясным 2 элементом, но, вызывает сомнения в 1 и 3.

Особенностью теорий данного типа, является совместное рассмотрение вопросов о том, что редуцировать и каким образом. Задача не явно формулируется следующим образом: «необходимо так определить сознание, чтобы способ его редукции был очевиден»

Физикалистски настроенные исследователи склонны использовать комплексный термин «психика», подразумевая под ним все разнообразие сознательной жизни. Сюда можно отнести память, способность к интроспекции и контролю, внимание, знание и самосознание и другие. Некоторые аспекты, например волю, физикалисты отбросят, объясняя это нерелевантностью понятия.

Крайней разновидностью физикализма является элиминативизм. Основные его представители Пол Черчленд, Ричард Рорти и Пол Фейерабенд. Данная программа объяснения сознания предполагает уточнение понятий так называемой «народной психологии». Постулируется, что некоторые элементы, исторически относящиеся к сознанию, являются просто ошибочными, такими же, как флогистон или импетус, другие же должны быть прояснены в терминах нейрофизиологии. Например, понятие памяти в терминах народной психологии представлялось единым непрерывным явлением. Нейрофизиология же предоставляет достаточно эмпирических фактов, чтобы сделать вывод, что память на самом деле 
комплексное явление состоящие из нескольких независимых типов активности мозга. Это подкрепляется рассуждениями о том, что «народная психология» фактически сама использует семантику, схожую с семантикой теоретической науки, но делает это стихийно. Задача исследователя обнаружить внутреннюю связь в обыденном языке и соотнести её со связью в языке теоретическом, тем самым уточнив термины для дальнейшего использования. Фактически такой подход предполагает, что психологические термины ошибочны, а психических состояний не существует.

Пол Черчленд описывает возможные следствия из этого соотнесения:

1) Народная психология вместе с ее онтологией будет полностью элиминирована, человечество вместо нее будет использоваться язык нейробиологии

или

2) Вместо народной психологии будет использоваться новый язык, в некотором смысле совпадающий с нашей врожденной концептуальной организацией.

Оба варианта развития событий являются пределом физикалисткого исследования, и, как кажется, скорее, указывают его слабые места, нежели радужные горизонты. Вариант 1, кажется нелепым, т.к. использование сложных объяснений в обыденной жизни противоречит здравому смыслу. Вариант 2 предполагает наличие врожденной концептуальной организации, что является спорной посылкой, т.к. ясно, что концептуальная организация врождена лишь отчасти, но так же приобретается в процессе жизни.

Очевидно, что эмпирическая наука действительно может уточнить некоторые обыденные понятия, связанные с сознанием, но кажется сомнительным то, что она может элиминировать их. Рассмотрим более подробно пример с теплородом, который любят приводить сами физикалисты. Данная концепция обладала всеми свойствами научной теории, она описывала явления, предсказывала результаты и т.д. до тех пор, пока наука не столкнулась с новыми явлениям и потребовалась новая теория. Таким образом, теплород был элиминирован в результате, говоря в терминах Т. Куна, накопления аномалий. На первый взгляд, кажется, что достаточно признать за некоторыми понятиями, связанными с сознанием 
статус аномалий, как они будут элиминированы. Но это явная ошибка. Элиминируются не сами аномалии, а связанные с ними объяснения. Аномалии требуют нового объяснения в новой теории и сохраняют свой онтологический статус в неприкосновенности. Показать же, что не только объяснение было не верным, но и сама аномалия была иллюзией или следствием этого объяснения такой анализ не в состоянии. Даже новая молекулярно-кинетическая парадигма, формально уничтожила теплород лишь благодаря своим эвристическим свойствам (передача тепла могла быть объяснена и без теплорода). Но из молекулярно-кинетической теории логически не следует отсутствие теплорода, ученые просто отказались от него за ненадобностью. С аспектами сознания подобная процедура не кажется возможной.

Чтобы сделать это более ясным, попробуем применить к понятиям, связанным с сознанием, бритву Оккама. Так уничтожается теплород или флогистон, т.к. те явления, для объяснения которых они были призваны, объективно объясняются и без них. Ключевым в этом объяснении является понятие объективности. Лишь объективное знание доступно критике [1], и как следствие, применению подобных принципов. Сознание же не обладает такой «бесплатной объективностью», многие исследователи предполагают, что субъективность его сущностное свойство.

С другой стороны следует признать, что элиминативистский подход задает верный вектор в исследовании сознания. Начинать такое исследование стоит с объективации понятий и их точного формулирования. Иначе теория в лучшем случае, будет интересным размышлением на тему, но не принесет ни согласия в научное сообщество, ни практической пользы.

Многие исследователи, столкнувшись с трудностями, которые не решал обычный редукционизм, решили смягчить некоторые его требования. Фактически редукция объясняет лишь нейронные процессы. Корреляция между психикой и этими процессами просто постулируется. Но от теории сознания в первую очередь требуется объяснить данную корреляцию. Одним из первых философов выдвинувших смягчающий принцип был Дональд Дэвидсон, с его концепцией применения понятия супервентности к сознанию. Супервентность Дэвид Чалмерс, развивший идеи Дэвидсона, определяет следующим образом: «набор свойств А супервентен по отношению к другому набору свойств В в том случае, когда два факта не могут иметь различий в отношении А-свойств, не имея такого же различия в своих В-свойствах» или «полное определение одного множества факта, другим множеством фактов» [2]. 
Сознание супервентно своему материальному носителю, т.е. с одной стороны полностью определяется им, но с другой не требует для себя ни дополнительной субстанции, ни собственных законов существования.

Чтобы прояснить термин супервентность, рассмотрим его рядом с каузальной связью. Каузальная связь двух объектов не предполагает, какой либо их онтологической зависимости между собой. Два бильярдных шара взаимодействуют, один влияет на другой при соударении, но последний существует независимо от первого. Можно сказать, что при взаимодействии шаров движение второго каузально возникает в результате движения первого.

Супервентность предполагает полную онтологическую зависимость объектов, а так же невозможность различия высокоуровневых свойств, при тождестве их низкоуровневых оснований. Так, например, химические факты супервентны на физических. Если исчезнут элементарные частицы, молекулы тоже исчезнут. Микрофизические свойства молекул воды полностью конституируют ее. Т.е. свойства жидкости супервентны на свойствах молекул. Но, тем не менее, очевидно, что свойства воды не тождественны свойствам молекул $\mathrm{H}_{2} \mathrm{O}$.

Таким образом, психическая реальность была реанимирована. Смягчив некоторые требования редукционизма и, избежав тем самым острых углов, данный подход воскресил старые проблемы в объяснении сознания. А именно связь между психическим и физическим миром.

Психофизическая проблема, в рамках концепции, формулируется и решается следующим образом:

1) Существует связь между ментальными и физическими событиями.

2) Физические события подчиняются строгим законам.

3) Ментальные события подчиняются не строгим законам.

4) Сознание супервентно своему физическому носителю, но формализовать связь между ментальным и физическим невозможно, ввиду характера ментальных законов. Дэвидсон в своей работе «Материальное сознание» пишет: «Легко понять тот факт, что, хотя каждое психологическое событие или состояние имеет физическое описание, это не дает нам оснований надеяться на то, что любой физический предикат вне зависимости от своей сложности имеет такой же объем, как и данный психологический предикат, и в меньшей степени следует надеяться на то, что существует физический предикат, законоподобным образом соотносящийся с данным психологическим предикатом», и там же «Если я прав, тогда детализированное знание физики или физиологии мозга, 
а в действительности всего человека в целом, не обеспечит упрощения интерпретации психологических понятий. Интерпретировать то, что l'homme machine имеет в виду, будет не легче, чем интерпретировать слова человека, и сама проблема не будет существенно иной» [3].

Данный подход ставит две, по существу разные, проблемы. Первая проблема является гносеологической, она может быть сформулирована следующим образом: «Каким образом относятся друг к другу термины разных уровней описания, например биологии и физики?» и вторая - онтологическая: «Каким образом сами макроявления, относятся к своим частям?».

Рассмотрим первую из них. Можно предложить два различных типа решения:

1) Единственная адекватная терминология - терминология физики, т.к. физическое знание фундаментально. Все остальные терминологии существуют «ради удобства» и являют собой либо неправомерные обобщения, либо эволюционные находки человечества для описания природы. Такой ответ подразумевает возможность редукции и сохраняет за физикой онтологический и гносеологический авторитет. Действительным объяснением сознания будет объяснение с помощью терминологии более низкого уровня реальности (физики, биологии и т.д.)

2) Каждый уровень описания является релевантным, т.к. ему соответствует собственный пласт объективной реальности. Высшие уровни реальности характеризуются свойствами, которые не могут быть выражены в терминологии низших уровней. Этот ответ подразумевает нередуцируемость, и, как кажется, опирается на один из законов диалектики - переход количественных изменений в качественные. Таким образом, удается усидеть на двух стульях. С одной стороны, изменения происходят только за счет изменения количества движения или материи, что сохраняет за физикой онтологический авторитет. С другой стороны для описания новых качеств, требуется новая терминология. Новые качества не следуют автоматически из старых.

Следует отметить, что второй ответ не подразумевает полного отсутствия редукции. Было бы нелепо утверждать, что, например, химическую реакцию нельзя описать в физических терминах. Лишь некоторая часть свойств, возникающих на новом уровне реальности, нередуцируема. Свойства двух атомов кислорода и свойства молекулы кислорода различаются кардинально. И, вероятно, свойства молекулы, не могут 
быть обнаружены, если в распоряжении у исследователя есть лишь разделенные атомы.

Вторая проблема так же предполагает два угла зрения, которые соответствуют решениям первой:

1) Редуктивный ответ, подразумевающий, что любой сложный объект есть прямая сумма своих частей. Никаких новых свойств в действительности не возникает, те свойства, которые мы обнаруживаем в объектах, лишь ужасающая по своему объему комбинация элементарных свойств.

2) Нередуктивный ответ. Его можно так же охарактеризовать как диалектический. При сложении свойств элементарных объектов, в согласии с законом диалектики, могут получаться принципиально новые свойства и способы взаимодействия.

Из комбинации решений первой и второй проблемы могут получаться различные направления исследования сознания. (1)+(1) = редуктивный физикализм, (2)+(2) = дуализм свойств, и последний, внутренне непротиворечивый вариант (2)+(1) = супервентный физикализм.

В целом концепция супервентности в изложении Дэвидсона имеет скорее критический, нежели позитивный характер. В дальнейшем идею супервентности разовьет и будет использовать в более продуктивном ключе Дэвид Чалмерс.

Следующим шагом в смягчении физикалистских установок является функционализм. Функционализм в философии сознания - доктрина, согласно которой нечто оказывается ментальным состоянием определенного типа не благодаря его внутренней конституции, но в зависимости от способа его функционирования или ролей, выполняемых им в системе, частью которой оно является.

Тем не менее, функциональное объяснение попадает в разряд редуктивных. Чалмерс, в своей работе «Сознающий ум» комментирует это следующим образом: «Редуктивное объяснение феномена не обязано требовать редукции данного феномена.... В известном смысле, феномены, которые могли быть реализованы на многих различных носителях например, научение, - могли бы и не быть редуцируемы в том смысле, что мы не можем отождествить научение с каким либо конкретным низкоуровневым феноменом. Но эта множественная реализуемость не препятствует редуктивному объяснению любых примеров обучения в терминах низкоуровневых феноменов»[4]. 
Методология функционального подхода может быть представлена следующим образом:

1) Придание понятию объекта специальной формы, которую можно редуцировать, путем определения каузальной роли.

2) Раскрытие механизмов действия этой роли.

Функционализм является подмножеством, так называемых теорий «от третьего лица». Считается, что для объяснения сознания не обязательно опираться лишь на свой субъективный опыт (от первого лица), но можно изучать психические состояния, поведение, а так же историю других субъектов.

Функциональный подход подразумевает рассмотрение сознания как конгломерата состояний нервной системы, каждое из которых выполняет определенную роль (функцию). Эти роли возникали эволюционно, постепенно образую сложную систему, взаимодействие элементов которой и порождает наше сознание[5].

Чтобы отличить сознательные процессы от всех остальных потребовались специальные теории, связывающие нейронные корреляты сознания с феноменологией. Некоторые исследователи считают, что сознательными являются те процессы, которые глобально доступны для всех когнитивных функций. Например, теория глобального рабочего пространства Бернарда Баарса, предполагает модульное строение сознание. Работа каждого модуля в отдельности не порождает сознания, но если какой то процесс доступен для всех модулей, то он становится сознательным. Другие, как Деннет, представляют сознание, системой конкурирующих процессов в мозге. Победитель в конкурентной борьбе попадает в «поле сознания». Данные объяснения, тем не менее, лишь отодвигают в тень вопрос о том, почему та или иная нейронная организация порождает сознание.

Сильной стороной функционального подхода является его опора на эмпирические данные. Функционализм по существу является философским обобщением уже полученных данных о работе и эволюции мозга и психики. При этом данная теория не подразумевает предсказаний. Мы не можем сделать из нее каких либо самостоятельных выводов о том, каким сознание будет в будущем, и она лишь с натяжкой может объяснить каким будет сознание в каких-то определенных условиях.

Двумя базовыми понятия функционализма являются «нейтральность функций» и «множественность реализации». Нейтральность означает, что сознательные состояния не являются ни материальными, ни 
ментальными. По существу такая формулировка должна снимать противопоставление материи и сознания, а так же все вытекающие из этого противопоставления проблемы. Исследование сознания приравнивается к исследованию нейтральных функциональных состояний и осуществляется по улучшенной бихевиористкой схеме: входные данные - функциональная обработка - выходные данные. Т.е. сознание представляет собой функцию, выполненную на каком либо физическом носителе, но не сводимую к нему. Остается не ясным, является ли «нейтральность» общим свойством всего мира, как предполагал Мах, или же это специфическая особенность функций. В любом случае это лишь отодвигает проблему на задний план.

Множественность реализации подразумевает независимость функции от физического носителя, на котором она реализуется. Из этого можно сделать вывод, что сознание может быть воплощено в машине, при наличии соответствующей программы. (Это допущение было определено как концепция сильного искусственного интеллекта).

Одним из аспектов, в рамках которого данный подход может подвергаться критике является понятие «смысла». Кажется, что человек обладает каким-то особым способом понимания, который нельзя обнаружить в функциональной структуре. Эта критика сконцентрирована в известном мысленном эксперименте «Китайская комната», предложенным Джоном Серлем. Суть его заключена в том, что субъект (например, человек) оперируя символами языка, который он не знает, по заранее заданным правилам, будет не осмысленно выполнять «правильные» действия. Тем самым демонстрируется, что помимо «правильного» действия в мире присутствует дополнительный компонент «смысл».

Данный эксперимент предполагает, предложенную Серлем концепцию двойной интенциональности - «исходной» и «производной» (здесь интенциональность можно понимать как «смысл»). Исходная интенциональность это та, которой обладает и может вкладывать в предметы человек, производная - та, которая зафиксированная в предметах. Т.е. в «китайской комнате» символы представляют собой носители производной интенциональности (другие люди вложили в них смысл), а ничего не понимающий человек призван демонстрировать отсутствие исходной.

Можно возразить на это отрицанием легитимности понятия «исходная интенциональность». Деннет в работе «Виды психики на пути к пониманию сознания» показывает, что в действительности существует только производная интенциональность. И тот «смысл», за которым, как нам кажется, стоит некоторая мистическая человеческая способность, на 
самом деле лишь производится некоторой сторонней системой и встраивается в нашу. Это «встраивание» подразумевает не более чем выполнение функциональной роли в нашей системе. Если нечто для нас бесполезно, не может быть использовано нашей системой (как китайский иероглиф), мы его не понимаем. Зато мы понимаем правила оперирования и можем следовать им для достижения результата. Таким образом, в китайской комнате возникает понимание лишь той части процесса, которая может быть встроена в функциональное устройство участников. Для стороннего наблюдателя китайца - это символы, для англичанина - это набор правил.

Более того, интенциональность может быть истолкована как не присущая объектам вообще, а лишь как инструмент описания данного объекта, находящийся в руках субъекта. Так решается в рамках данного подхода проблема других сознаний. Нам не нужно постулировать или каким либо образом обнаруживать сознание у других. Просто нам удобно приписывать им сознание для описания их поведения. И те же рассуждение можно применить в отношении машин, или искусственного интеллекта. Если мы не сможем описать их деятельность в логических терминах, нам придется приписать им наличие сознания.

Тем не менее, остается не ясно, каким образом конкретная функциональная организация, пусть и сложная, порождает сознание. В любом случае такая организация должна быть воплощена на некотором носителе, представим, что это процессоры связанные проводами. Пускай их организация, почти полностью повторяет наш мозг в каком-либо его состоянии и имеется достаточно устройств ввода информации. Система будет переходить в новые функциональные состояния с течением времени по очевидным законам, но ни сами состояния, ни переход от одного к другому не предполагают возникновения той ясности, которую мы называем сознанием. Кажется, что главная ошибка функционализма (если это ошибка), состоит в отождествлении понятий мышления и сознания. Сознание фактически признается частным случаем мышления, самым развитым и сложным случаем, возникшим в результате эволюции и под воздействием культуры. Многие философы не приняли такое объяснение сознания, считая его лишь объяснением мышления. Томас Нагель в работе «Что значит быть летучей мышью?» пишет: «Это ощущение может принимать различные формы, и возможно (хотя я в этом сомневаюсь), что разница форм повлияет и на разницу в поведении. Но в общем можно сказать, что некий организм впадает в сознательное состояние только тогда, когда быть этим организмом на что-то 
похоже - для него самого. Мы можем называть это субъективным характером опыта. Это качество нельзя уловить при помощи хорошо знакомых, недавно разработанных методов редукционного анализа феноменов мышления, поскольку все эти методы логически совместимы с его отсутствием».

Подведем итог, противники функционализма указывают на объяснительный разрыв в материалистическом объяснении психики. Функциональное объяснение по своему принципу повторяет любое научное объяснение - достаточно указать каузальную роль явления, тем самым получив всю возможную информацию о нем. При этом кажется, что сознательные явление претендуют на дополнительное объяснение - ответ на вопрос «чем данный феномен является для меня», и каким образом возникает это «для меня». Сами функционалисты считают данные вопросы нелегитимными, ошибками в постановке проблемы. Как следствие из этой конфронтации между «школами» практически не возникает продуктивного диалога, так как разногласия содержатся в самом корне воззрений, первое из которых можно назвать научным, второе метафизическим.

Фактически редуктивные теории объясняют то, как психическая жизнь устроена, но не объясняют сознание само по себе. В этом состоит главный аргумент антиредукционистов - сознание не логически необходимо сопровождает функциональную работу мозга. В отличие от экстерналистких редуктивных теорий, их противники опираются на интерналистскую эпистемологию, предполагающую рассмотрение данных от первого лица.

Казалось бы, способ рассмотрения мира с позиции третьего лица зарекомендовал себя как единственно верная позиция в познании, но сознание является специфическим объектом. Смотреть на мир со стороны можно до тех пор, пока в поле зрения не попадает сам смотрящий. Ухищрения в попытках рассмотрения сознания с позиции третьего лица не приводят к желаемому результату, так как, во-первых, сознание само является основой всякого другого знания, во-вторых, в каждом акте познания содержится не только элемент объекта познания, но и субъекта. Таким образом, при рассмотрении сознания «со стороны» теряется субъективная составляющая, которая для понимания сознания является решающей. Сторонники теорий от первого лица указывают на объяснительный разрыв, возникающий при рассмотрении сознания классическим для науки способом. 
Если определить сознание, вслед за Томасом Нагелем, следующим образом: «в общем можно сказать, что некий организм впадает в сознательное состояние только тогда, когда быть этим организмом на что-то похоже - для него самого», то редуктивные объяснения действительно не касаются сознания. Факты о переживании организма доступны только ему самому, но никогда стороннему наблюдателю. Невозможно объяснить, по крайней мере, со стороны, почему функциональные или нейробиологические процессы сопровождаются субъективным опытом. Рассмотрим аргументацию в пользу объяснительного разрыва:

1) Макроскопическое явление можно редуктивно объяснить, если существует априорное следование этого явления из микрофизических фактов.

2) Априорное следование обеспечивается анализом понятия в терминах его функции.

3) Если имеет место априорное следование, то макроявление логически необходимо присутствует, когда реализуется соответствующая микрофизическая конфигурация.

4) Функциональный анализ всегда оставляет возможность мыслить любую конфигурацию без субъективного опыта.

5) Субъективный опыт не является априорным следствием функционального объяснения.

6) Существует объяснительный разрыв.

Т.е. по существу вся аргументация против редуктивного объяснения стоит на двух китах: признания за сознанием особого статуса и недостаточности эмпирических корреляций между сознанием и его проявлениями для объяснения. «Но чистое, никак не объясненное сопутствие - это не решение, а знак того, что здесь имеет место нечто фундаментальное, чего мы не знаем....Мы не можем рассматривать чистое сопутствие как конец дела, потому что это означало бы признать, что физическое делает необходимым ментальное, никак не отвечая на вопрос, как оно это делает»[6].

Можно возразить:

1) Если на текущий момент не существует редуктивного объяснения, из которого бы a priori следовало сознание, то из этого нельзя заключить, что такого объяснения в принципе не существует. 
2) Разрыв обусловлен не онтологической особенностью сознания, но лишь использованием специфических феноменологических понятий, не сводящихся к понятиям от третьего лица.

Ясно, что разногласия базируются на разных интерпретациях сознания. Глубокая убежденность в особенности субъективного опыта у одних, и столь же глубокое отрицание этой особенности коренятся в онтологических основаниях каждой группы исследователей. Первые склонны дуализму, вторые верные последователи материального монизма. При такой ангажированности, возможно лучшим способом рассуждения о сознании будет вариант Д. Сёрля: «Вывод отсюда такой: как только вы поймете противоречивость дуализма, вы также сможете понять, что материализм и монизм столь же ошибочны. Дуалисты спрашивают: "Сколько имеется видов вещей и свойств?" и считают до двух. Монисты, поставленные перед тем же вопросом, не идут дальше одного. Но подлинная ошибка заключалась в том, что вообще начинали считать. Монизм и материализм определяются в терминах дуализма и ментализма, и поскольку определения ментализма и дуализма противоречивы, то монизм и материализм наследуют эту противоречивость». Противоречивым кажется само понятие субстанции. Множественность субстанций требует либо предустановленной гармонии, либо божественного вмешательства, а одной субстанции не хватает для объяснения феноменов сознания.

Стратегия «познай самого себя» имеет ряд существенных недостатков. Она, как правило, не обладает всеми основополагающими критериями знания: фальсифицируемостью, проверяемостью, воспроизводимостью. Явления субъективной реальности уникальны и выявление инвариантов этих явлений должно стать приоритетным направлением в области исследования сознания. Франц Брентано был одним из первых, кто обратил на это внимание: «Интенциональные отношения в созерцаниях с психическим содержанием обнаруживают столь же многообразные различия, что и чувственные качества в созерцаниях с физическим содержанием репрезентации. И как там, на основании глубоких различий чувственных качеств устанавливается количество чувств, так и здесь на основании глубоких различий интенциональных отношений определяется количество классов основных психических феноменов». 
Сам Брентано опирался на различения, найденные еще Декартом: представления, суждения, эмоции. Истинность физических содержаний определялась посредством суждений, психически - с помощью эмоций.

Другой способ определения инвариантов предложен одним из современных теоретиков сознания Дубровским Д.И.[7]:

1) Первичное описание явлений субъективной реальности.

2) Формирование личностного инварианта явления, придание данному содержанию сознания диспозиционного статуса.

3) Формирование межличностного инварианта.

Данная схема является большим приближением, но указывает верное направление исследования. Для уточнения этой схемы требуется определить механизм формирования данных инвариантов, т.к. при произвольном выборе возникает проблема валидации. Нет причин предпочесть один из противоречащих друг другу инвариантов, если они получены разным путем.

Смысл инвариантности в том, что нечто сохраняется лишь при определенного вида преобразованиях. Род этих преобразований и требуется установить. Возможно, для этого потребуется предварительно осуществить категоризацию сознательных явлений, а затем в каждой из категорий определить вид преобразований. Например, эмоциональное переживание боли имеет ряд характеристик (интенсивность, продолжительность), но каждое отдельное переживание имеет общие черты (которые в совокупности и названы неточным термином боль).

Эпистемология сознания развивается по диалектическому закону единства противоположности. Теории от первого лица противопоставлены теориям от третьего, но на текущий момент начинается осуществляться синтез данных воззрений, который снимет внутренние противоречия каждого подхода и, возможно, поможет пролить свет на проблему сознания.

Хорошей основой для такой эпистемологии является «теория экологического восприятия» Дж. Гибсона. В ней Гибсон исходит из того факта, что явления для живого существа и явления природы сами по себе разных порядков. Мир вокруг человека или животного обладает собственными свойствами, которые можно, но бессмысленно описывать в физических терминах.

«Движение предметов в окружающем мире представляет собой явление другого порядка, нежели движение тел в пространстве»- Дж. Гибсон «Экологическая теория восприятия» [8]. 
Экологический мир (окружающий мир субъекта) обладает двумя свойствами, иерархичностью и значимостью. Иерархичность означает, что объекты встроены друг в друга в пространстве и во времени. Иерархичность призвана заменить геометрическое понятие взаимного расположения. Значимость мира передается Гибсоном с помощью термина «возможность» - то, что объект предоставляет субъекту. Стоит обратить внимание на то, как субъективистский термин «значение» переходит в объективистский термин «значимость». Если мир рассматривается как совокупность физических объектов, то значение является искусственным дополнением. Если же встать на экологическую точку зрения, значение можно обнаружить, что делает его объективным.

При движении по шкале теорий сознания в сторону единицы, существенно изменяются не только гносеологические подходы, но и онтология. Онтологическим основанием редуктивных теорий является материализм. Данное основание является первой целью критики философов относящихся к сознанию «серьезно». Аргумент против материализма может быть выражен следующим образом: сознание не является логически супервентным своему физическому носителю, так как мыслим мир, физически идентичный нашему, но в котором отсутствует факт сознания. Значит, сознание является дополнительным по отношению к физической реальности, следовательно, материализм ложен. Данный аргумент может отрицаться с трех позиций:

1) На самом деле мир, тождественный нашему во всех отношениях, кроме факта сознания, не мыслим.

2) Даже если такой мир мыслим, из этого не следует его физической возможности.

3) Тождественный мир без сознания мыслим лишь потому, что нам не хватает знания о нашем мире, чтобы обнаружить явное противоречие.

Если мыслимость подразумевает отсутствие противоречия, то сторонники контраргумента (1) должны указать на него. Действительно, если сознание является чем то реальным, то мы можем обнаружить его в мире. Оно играет в нем каузальную роль. Но тогда из тождественности мира, в котором присутствует сознание и мира, в котором сознания нет, следует, что сознание не играет никакой каузальной роли в мире и значит, вообще не существует. Этот аргумент верен, если верна каузальная теория значения.

Второй контраргумент подкрепляется существованием необходимо априорных истин (С. Крипке «Именование и необходимость».). Класси- 
ческим примером такой истины является «Вода есть $\mathrm{H}_{2} 0$ ». Мыслимо, что вода не является $\mathrm{H}_{2} \mathrm{O}$, но метафизически это невозможно.

Кажется, что первый контраргумент более сильный, т.к. опирается на более ясную теорию. Второй же можно обойти, используя двумерную модель апостериорной необходимости. Утверждается, что любому понятию соответствует два интенсионала. Первичный или априорный и вторичный - апостериорный. Чалмерс, предлагает сфокусироваться на первичном интенсионале.

Третья стратегия: подразумевается отсутствие на текущий момент знания, позволяющего указать, как связано сознание и физические процессы. Такое знание может быть обнаружено либо в области фундаментальных законов, либо в области макроявлений. Будущие открытия в одной из областей могут пролить свет на проблему.

В целом аргумент мыслимости кажется более чем спорным. Например, на первый взгляд, мыслима скорость, превосходящая скорость света, не смотря на то, что физически это невозможно. Каким образом можно представить данный процесс? Рассмотрим два достаточно удаленных объекта в пространстве, например Солнце и Землю. Далее представим объект и мысленно перенесем его от Земли к Солнцу за мгновение. Зная, что свет проходит это расстояние за 8 минут, мы, якобы, получили необходимо представление. Ошибка здесь кроется в возможности представления двух удаленных в пространстве объектов. Мы конечно можем представить, что находимся в галактике Андромеда, и расстояние между Землей и Солнцем для нас несколько миллиметров, но и свет пройдет такое расстояние невероятно быстро. Представить же расстояние галактического порядка без перспективы невозможно.

Аргумент мыслимости скорее преследует цель убедить оппонентов, нежели является самостоятельным основанием дуалистического взгляда. В корне «серьезного подхода к сознанию» лежит не мыслимость миров без сознания, а субъективный опыт конкретного философа. Субъективный опыт в любой дискуссии не является серьезным аргументом, но специфика сознания, кажется, дает исследователям право на его использование. Основным локомотивом дуалистического направления современной философии является Дэвид Чалмерс. Он детализирует понятие супервентности, разделяя ее на логическую и естественную. (Чалмерс, сознающий ум). Логическая супервентность подразумевает, что не существует логически возможных ситуаций, тождественных в низкоуровневых свойствах, но различных в высокоуровневых. Естественная супервентность подразумевает, что во всех естественно воз- 
можных ситуациях определенные низкоуровневые свойства конституируют определенные высокоуровневые. Сознание является естественно, но не логически супервентным на физическом. В результате для сознания не требуется собственной субстанции, отличной от материи, при этом сознание оказывается фундаментальным явлением, нередуцируемым к физическому. При этом возникает две трудности: так как свойства сознания невозможно редуцировать, не ясно, каким образом их можно описать.

Раз сознание обладает специфичными фундаментальными свойствами, значит по аналогии с физикой, существуют и фундаментальные законы. Стратегия исследования в таком случае будет состоять в определении этих свойств и обнаружении законов.

Критика аргумента логической представимости показывает, что сознание в рамках такого подхода оказывается эпифеноменом (не играет каузальной роли в мире).

Ответом на это может быть отсылка к реляционной структуре физического знания. Действительно, физические характеристики объекта всегда говорят лишь об его отношении к другим объектам в разных процессах. Обобщая, мы получаем картину мира как каузального потока. При этом о собственных свойствах того, что включено в этот поток физическая теория умалчивает. Лишь свойства сознания являются свойствами «вне отношений», но присущими объекту самому по себе. Естественно предположить, что и у физических объектов должны быть подобные внутренние свойства, которые конституируют внешние.

Таким образом, аргумент логической представимости сохраняет каузальную роль сознания, т.к. можно предположить, что представляя физически идентичный мир, но без фактов сознания, мы в действительности подменяем либо игнорируем внутренние (феноменальные) свойства физического.

Нас вполне устраивает описание электрона как сущности порождающей электрический ток и обуславливающей химические реакции. Вопрос о том, почему электрон ведет себя именно так, кажется нелепым. Даже Гегель критически относился к попыткам ответа на вопрос «почему?» в физической реальности: "Лучше сказать, что магнит (как выражается Фалес) имеет душу, чем, что он имеет силу притягивать; сила - это такое свойство, которое как отделимое от материи представляет себя в виде предиката, душа же- это движение себя, одно и то же вместе с природой материи". 
На текущий момент в научной и философской среде нет консолидации мнений относительно природы и свойств сознания. Одни исследователи пытаются рассмотреть сознание как очередной объект материального мира, другие требуют ответа на вопрос, почему этот объект обладает субъективным опытом. У всех концепций сознания совпадает предмет исследования, но различается метод.

Отказавшись от метафизического понятия субстанции, философ остается один на один с эмпирическим фактом сознания и вопросом «почему данная физическая организация порождает сознание?». Можно, вслед за Лейбницом, дедуктивно наделить сознательными свойствами все элементы реальности. Противоположная стратегия заключалась бы в том, чтобы попытаться ответить на вопрос «почему» для простых элементов и лишь затем индуктивно получить решение проблемы сознания.

Но ни современная наука, ни философия не располагают инструментами для решения подобных проблем. Все взаимодействия, начиная от квантово-механических частиц, до социальных волнений лишь постулируется, внутренние причины - исток этого взаимодействия, остаются скрытыми.

\section{Примечания}

1. Попер Карл Р. Объективное знание. Эволюционный подход. Пер. с англ. Д.Г. Лахути. - М.: Эдиториал УРСС, 2002. - С. 24-26.

2. Чалмерс Д. Сознающий ум: в поисках фундаментальной теории. - М.: УРРС: Книжный дом «Либроком», 2013. - С. 125-127.

3. Дэвидсон Д. Материальное сознание. Аналитическая философия: Избранные тексты. - М.: Изд-во МГУ, 1993.

4. Чалмерс Дэвид. Сознающий ум: в поисках фундаментальной теории.- М.: УРРС: Книжный дом «Либроком», 2013. - С. 29.

5. Деннет Дэниел. Виды психики: на пути к пониманию сознания. Перевод с англ. А. Веретенникова. - М, Идея-Пресс, 2004.

6. Нагель Томас. Мыслимость невозможного и проблема духа и тела // Вопросы философии. - 2001. - № 8 .

7. Дубровский Д.И.. Информационный подход к проблеме «сознание и мозг» // Вопросы философи. - 1976. - № 11 .

8. Гибсон Дж. Экологический подход к зрительному восприятию // М.: Прогресс, 1988. 